Renal Medicine

\author{
Edited by Dr CRV Tomson FRCP Consultant Renal Physican \\ Richard Bright Renal Unit, Southmead Hospital, Bristol
}

characteristic finding in Wegener's granulomatosis, microscopic polyangiitis and Churg-Strauss syndrome. The discovery of antineutrophil cytoplasmic antibodies (ANCA) in the blood of patients with pauci-immune small vessel vasculitis has been an extremely important advance in the diagnosis of these conditions.

\section{Small vessel vasculitis}

\section{Multisystem diseases and the kidney}

Wendy Metcalfe MRCP, Specialist Registrar in Renal Medicine

\section{Christopher Dudley MD FRCP, Consultant} Renal Physician

Richard Bright Renal Unit, North Bristol NHS Trust, Southmead Hospital, Bristol

\section{Clin Med JRCPL 2002;2:188-91}

It is difficult to name a multisystem disease that does not in some way affect the kidneys either directly as part of the disease process or as a result of treatment. In this review, 'multisystem diseases' are taken to mean those conditions characterised by systemic vasculitis unless otherwise specified. These rare diseases may present to almost any hospital physician, frequently with non-specific signs and symptoms, usually associated with an acute phase response (raised C-reactive protein (CRP) concentration, erythrocyte sedimentation rate (ESR) or plasma viscosity). Recognition of renal involvement by the detection of blood and protein on urine dipstick, even in the presence of a normal creatinine, provides both a clue to multisystem involvement and an early diagnostic opportunity with a renal biopsy.

\section{Systemic vasculitis}

Diseases in which vasculitis is a primary process are known generically as primary systemic vasculitides. The main types can be described using clinical features and histological findings based on the size of blood vessel involved, as agreed by expert consensus (Chapel Hill Consensus Conference) (Fig 1) ${ }^{1}$. Vasculitis may also occur as a secondary feature in other diseases, such as systemic lupus erythematosus and rheumatoid arthritis (RA). Renal involvement is most common in small vessel vasculitis, perhaps because the kidneys provide a rich capillary (glomerular) bed. Vasculitis of the glomeruli is recognised histologically as a necrotising glomerulonephritis, often with associated crescents due to the accumulation of cells and fibrin in Bowman's space. Immunohistochemical staining allows further categorisation. For example, the identification of glomerular immunoglobulin (Ig) A deposits in a patient with renal impairment, joint pain and a rash would be characteristic of Henoch-Schönlein purpura. More often, however, there is a paucity or absence of glomerular immune deposits and the lesion is referred to as 'pauci-immune'; this is the

\section{Presentation and diagnosis}

Small vessel vasculitis is increasingly frequently recognised due to increased awareness and availability of ANCA testing, but it remains an uncommon disease with an annual incidence of up to 20 cases per million population, the incidence increasing with age $\mathrm{e}^{1,2}$. Presenting symptoms are often non-specific; therefore, there is significant delay and organ damage may occur before the diagnosis is made. Differentiation of the various small vessel vasculitides, as defined at Chapel Hill, is not straightforward because there is a great deal of overlap in symptomatology and clinical features. In addition, the clinical picture of each syndrome varies considerably between patients and may also change in any individual through the course of their illness.

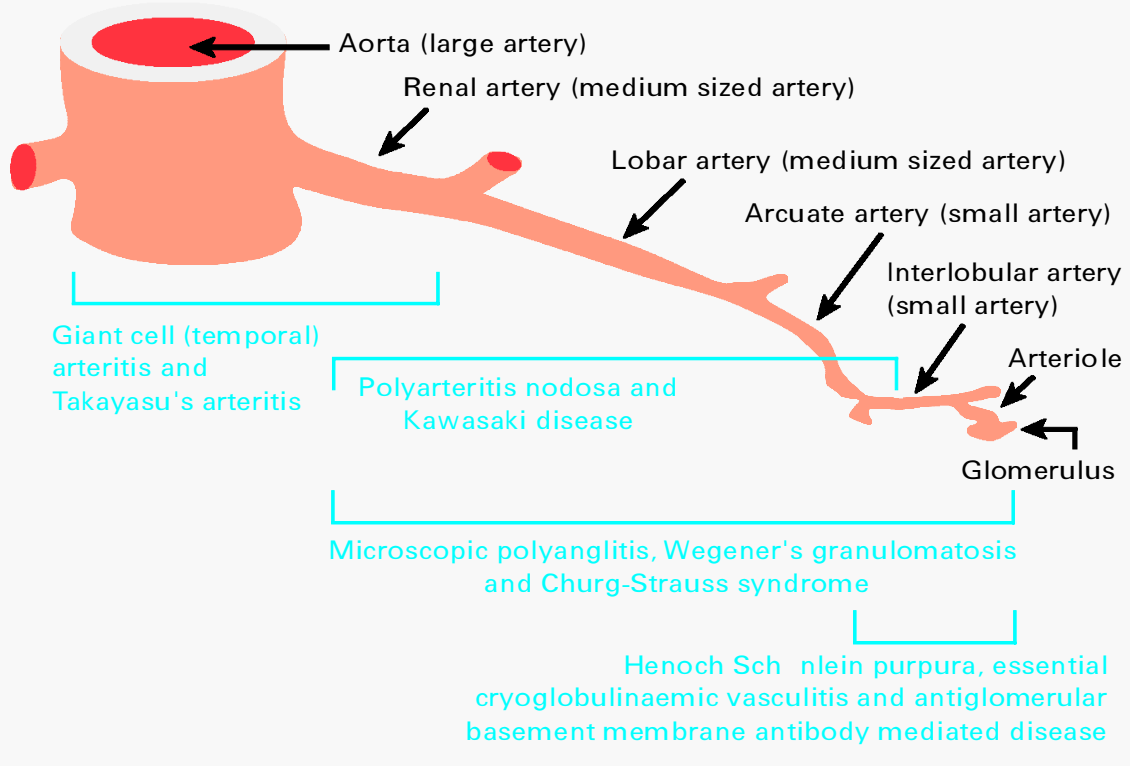

Fig 1. Spectrum of systemic vasculitides organised according to predominant size of vessels affected (reproduced, with permission, from Ref 1 ). 


\section{Small vessel vasculitis associated with antineutrophil cytoplasmic antibody}

Wegener's granulomatosis and microscopic polyangiitis. Wegener's granulomatosis and microscopic polyangiitis share many features, but granulomatous involvement of upper and lower airways as well as extrapulmonary granulomata are specific to Wegener's granulomatosis (Table 1). Epistaxis, rhinorrhoea, nasal discomfort, facial pain, otitis media and conductive hearing loss, hoarseness and haemoptysis may all occur. In the lungs, parenchymal necrosis, vasculitis and granulomata may cause dyspnoea, cough, haemoptysis and chest pain. Non-specific symptoms, such as malaise, weight loss, fever, arthralgia and myalgia are common in both diseases and are often the first reported symptoms $\mathrm{s}^{3-5}$. Eye involvement is frequent, with episcleritis the most usual ocular manifestation. Necrotising vasculitis may also affect the brain and peripheral nerves, gastrointestinal tract, alveolar capillaries and, rarely, the heart.

Renal involvement is common, but rarely the earliest feature. The presence of microscopic haematuria and proteinuria are important pointers of renal involvement and should be actively sought. More advanced renal involvement results in impaired renal function, which may deteriorate rapidly. Renal biopsy shows a focal segmental necrotising glomerulonephritis, often with crescent formation, while immunohistochemistry is pauci-immune. Vasculitis of the interlobular arteries and arterioles may be found.

Diagnosis of Wegener's granulomatosis and microscopic polyangiitis requires histological proof of a small vessel vasculitis and, in the former, granulomatous inflammation in the respiratory tract. Blood tests may demonstrate a normochromic normocytic anaemia, neutrophilia, thrombocytosis, elevated CRP, ESR, plasma viscosity, alkaline phosphatase and hypoalbuminaemia. Serum urea and creatinine may be elevated but levels within the normal range do not exclude renal involvement. Positive ANCA when there is clinical

Table 1. Wegener's granulomatosis and microscopic polyangiitis.

\begin{tabular}{|c|c|c|}
\hline Feature & $\begin{array}{c}\text { Wegener's } \\
\text { granulomatosis }\end{array}$ & $\begin{array}{l}\text { Microscopic } \\
\text { polyangiitis }\end{array}$ \\
\hline Malaise, weight loss, fever & $\checkmark$ & $\boldsymbol{r}$ \\
\hline Myalgia, arthralgia & $\checkmark$ & $\checkmark$ \\
\hline Proteinuria, haematuria & $\checkmark$ & $\checkmark$ \\
\hline Renal failure & $\checkmark$ & $\checkmark$ \\
\hline Neurological involvement & $\checkmark$ & $\checkmark$ \\
\hline Gut involvement & $\checkmark$ & $\checkmark$ \\
\hline Cardiac involvement & $\checkmark$ & $\checkmark$ \\
\hline Ocular involvement & $\checkmark$ & $\checkmark$ \\
\hline $\begin{array}{l}\text { Upper and lower } \\
\text { respiratory tract involvement }\end{array}$ & $\checkmark$ & $\checkmark$ \\
\hline Upper airways granulomata & $\checkmark$ & $\boldsymbol{*}$ \\
\hline Lower airways granulomata & $\boldsymbol{\sim}$ & $\boldsymbol{*}$ \\
\hline Extrapulmonary granulomata & $\checkmark$ & $\boldsymbol{*}$ \\
\hline cANCA staining $^{1 *}$ & $\checkmark$ & $\boldsymbol{*}$ \\
\hline pANCA staining ${ }^{2 *}$ & $\boldsymbol{*}$ & $\checkmark$ \\
\hline
\end{tabular}

suspicion of vasculitis adds significant weight to the diagnosis, particularly if directed against proteinase 3 or myeloperoxidase by ELISA ${ }^{6,7}$.

Churg-Strauss syndrome. Respiratory involvement in Churg-Strauss syndrome, manifesting as nasal polyps, allergic rhinitis and late-onset asthma, often pre-dates vasculitis by years. Eosinophilia is a prominent feature ${ }^{8}$. An association with the use of leukotriene receptor antagonists has been reported, although causality is unproven. Vasculitic manifestations mainly affect heart, skin, bowel, muscle and the nervous system (mononeuritis multiplex). Renal disease, when present, is usually a pauci-immune focal segmental glomerulonephritis, and renal failure is uncommon. Patients may have a positive pANCA, usually with myeloperoxidase specificity.

\section{Small vessel vasculitis without antineutrophil cytoplasmic antibody}

Mixed essential cryoglobulinaemic vasculitis. Vasculitis develops when cryoglobulins deposit in blood vessel walls. The mixed cryoglobulin consists of a monoclonal IgM rheumatoid factor complexed to polyclonal IgG which readily activates complement, hence levels of C3 and C4 are reduced. Mixed essential cryoglobulinaemia is due to hepatitis $\mathrm{C}$ virus infection in over $80 \%$ of cases $^{9}$. Clinical features include purpura, arthralgia, leg ulcers, neurological manifestations and renal involvement. Typical findings on renal biopsy are a membranoproliferative glomerulonephritis with intraglomerular deposits.

Henoch-Schönlein purpura. HenochSchönlein purpura occurs most commonly in childhood but may occur at any age. It has a characteristic rash, initially urticarial but becoming purpuric, typically on limb extensor surfaces, ankles and buttocks. It is accompanied by joint pain, abdominal symptoms and renal disease. Generally, the clinical picture is diagnostic but the consistent finding of IgA deposition on renal biopsy differentiates it from other causes of small vessel vasculitis.

Classical polyarteritis nodosa. Polyarteritis nodosa is characterised by a necrotising vasculitis of medium-sized arteries, leading to aneurysm formation, tissue infarction, haemorrhage and 
Key Points

The finding of microscopic haematuria in patients with non-specific symptoms, signs and laboratory findings may point to a multisystem disorder

A normal plasma creatinine concentration does not exclude significant renal disease

Kidney involvement is common in small vessel vasculitis and provides an accessible organ from which to make a specific tissue diagnosis

Cholesterol crystal embolisation may mimic many of the features of small vessel vasculitis but can be distinguished histologically

KEY WORDS: CPD, cholesterol crystal embolisation, multisystem disease, necrotising glomerulonephritis, rapidly progressive glomerulonephritis, renal disease, systemic vasculitis, vasculitis

organ dysfunction. Clinically, fever, malaise, weight loss and symptoms from the gastrointestinal tract, nervous system, muscles and soft tissues are typical. Renal involvement may present as haematuria, loin pain or renal impairment due to renal infarction or ischaemia.

The diagnosis is best made by arteriography demonstrating medium-size vessel disease. Renal biopsy is usually not diagnostic unless a medium-size artery is included fortuitously in the specimen. Glomeruli are normal, and ANCA is usually negative.

\section{Large vessel vasculitis}

Renal involvement in large vessel vasculitis is rare. Cases of typical giant cell temporal arteritis with coexistent focal necrotising glomerulonephritis have been reported, as has temporal artery disease in patients with Churg-Strauss syndrome and Wegener's granulomatosis. The main renal arteries may be involved in Takayasu's arteritis ${ }^{10}$ but glomerular disease is extremely rare.

\section{Other multisystem diseases with renal involvement}

\section{Systemic lupus erythematosus}

Histological evidence of lupus nephritis is found in the vast majority of patients, even in the absence of clinical manifestations of renal disease ${ }^{11}$. Renal involvement is frequent, developing in the first three years of disease in up to $75 \%$ of patients. Clinical features are variable, ranging from asymptomatic proteinuria and haematuria to the nephrotic syndrome and acute renal failure. The renal biopsy findings and clinical features determine the type of therapy indicated. For monitoring disease activity in lupus nephritis, serial measurement of anti-C1q antibody levels may be more useful than $\mathrm{C} 3$ and $\mathrm{C} 4$ levels ${ }^{12}$.

\section{Scleroderma}

Approximately $50 \%$ of patients with scleroderma have some sign of renal dysfunction such as proteinuria, a mild rise in plasma creatinine or hypertension. More severe disease presents with rapidly progressive acute renal failure, accompanied by accelerated hypertension and a microangiopathic haemolytic anaemia (scleroderma renal crisis). Important risk factors include diffuse skin involvement, particularly if rapidly progressive, and high-dose corticosteroid use. Renal biopsy shows the characteristic 'onion skin' changes in the walls of small arteries and arterioles. Early use of angiotensinconverting enzyme inhibitors can prevent progression of renal failure ${ }^{13}$.

\section{Rheumatoid arthritis}

In RA, renal disease may be due to:

- the nephrotoxicity of drug treatment (non-steroidal anti-inflammatory drugs, penicillamine, gold)

- glomerular deposition of amyloid protein (AA amyloidosis)

- an associated proliferative glomerulonephritis.
Renal vasculitis complicating RA is rare; it causes a focal segmental necrotising glomerulonephritis identical to that seen in the other small vessel vasculitides and may be associated with the presence of $\mathrm{pANCA}^{14}$.

\section{Cholesterol crystal embolisation}

When atheromatous plaques ulcerate and become denuded of their endothelial covering (usually following vascular surgery or arteriography) the underlying cholesterol-rich extracellular matrix may become detached and embolise as numerous small particles composed principally of cholesterol crystals. With showers of emboli, tissue damage results. The lower limbs, gastrointestinal tract and kidneys are most frequently involved. The clinical features (hypocomplementaemia, eosinophilia, acute phase response and, rarely, positive ANCA) may result in a misdiagnosis of small vessel vasculitis and the inappropriate use of powerful immunosuppressive drugs ${ }^{15}$. Diagnosis of cholesterol crystal embolisation is by the histological demonstration of characteristic biconvex, needle-shaped cholesterol clefts within the lumen of arteries or arterioles of affected tissues or organs.

\section{References}

1 Savage CO, Harper L, Cockwell P, Adu D, Howie AJ. ABC of arterial and vascular disease: vasculitis. Review. $\mathrm{Br}$ Med J 2000; 320:1325-8.

2 Garrett PJ, Dewhurst AG, Morgan LS, Mason JC, Dathan JR. Renal disease associated with circulating antineutrophil cytoplasm activity. QJM 1992;85:731-49.

3 Pinching AJ, Lockwood CM, Pussell BA, Rees AJ, et al. Wegener's granulomatosis: observations on 18 patients with severe renal disease. QJM 1983;52:435-49.

4 Savage CO, Winearls CG, Evans DJ, Rees AJ, Lockwood CM. Microscopic polyarteritis: presentation, pathology and prognosis. QJM 1985;56:467-83.

5 Noritake DT, Weiner SR, Bassett LW, Paulus HE, Wesibart R. Rheumatic manifestations of Wegener's granulomatosis. J Rheumatol 1987;14:949-51.

6 Van der Woude FJ, Ferrario F. Renal involvement in ANCA-associated systemic vasculitis. J Nephrol 1999;12:105-28.

7 Falk RJ, Jennette JC. ANCA small-vessel 
vasculitis. Review. J Am Soc Nephrol 1997; 8:314-22.

8 Lanham JG, Elkon KB, Pusey CB, Hughes GR. Systemic vasculitis with asthma and eosinophilia: a clinical approach to the Churg-Strauss syndrome. Review. Medicine (Baltimore) 1984;63:65-81.

9 Sinico RA, Ribero ML, Fornasieri A, Renoldi $\mathrm{P}$, et al. Hepatitis $\mathrm{C}$ virus genotype in patients with essential mixed cryoglobulinaemia. QJM 1995;88:805-10.

10 Hall S, Barr W, Lie JT, Stanson AW, et al. Takayasu arteritis. A study of 32 North American patients. Medicine (Baltimore) 1985;64:89-99.

11 Bianco N, Vargas-Arenas R, Tapanes F, et al. Silent nephritis in systemic lupus erythematosus (SLE). Scand J Immunol 2001; 54(Suppl 1):100.

12 Moroni G, Trendelenburg M, Del Papa N, Quaglini S, et al. Anti-C1q antibodies may help in diagnosing a renal flare in lupus nephritis. Am J Kidney Dis 2001;37:490-8.

13 Steen VD, Costantino JP, Shapiro AP, Medsger TA. Outcome of renal crisis in systemic sclerosis: relation to availability of angiotensin converting enzyme (ACE) inhibitors. Ann Intern Med 1990;113:352-7.

14 Harper L, Cockwell P, Howie AJ, Michael J, et al. Focal segmental necrotizing glomerulonephritis in rheumatoid arthritis. QJM 1997;90:125-32.

15 Peat DS, Mathieson PW. Cholesterol emboli may mimic systemic vasculitis. $\mathrm{Br}$ Med J 1996;313:546-7.

\section{Cardiovascular}

\section{risk factors in}

\section{progressive}

\section{renal disease}

KPG Harris MA MD FRCP, Reader and Honorary Consultant Nephrologist, Leicester General Hospital

\section{Clin Med JRCPL 2002;2:191-4}

Cardiovascular disease (CVD) is one of the leading causes of mortality and morbidity in the western world. Epidemiological studies have identified important physiological and lifestyle risk factors for CVD (Table 1) ${ }^{1}$. More encouragingly, evidence from a number of randomised controlled trials and meta-analyses has now demonstrated that this disease may largely be prevented (at least in the general population) by lifestyle modification and drug treatment of risk factors ${ }^{2}$. However, cardiovascular risk management remains suboptimal, particularly in populations with certain chronic metabolic disorders such as chronic renal failure (CRF).

\section{Chronic renal failure and cardiovascular disease}

With modern nephrological management, the survival of patients with CRF has been greatly improved. Patients now survive their renal disease only to die prematurely of the complications of atherosclerosis, the most frequent cause of death in patients with end-stage renal disease $^{3}$. Cardiovascular morbidity and mortality are both several-fold higher in patients with CRF than among the general population: for example, cardiac mortality in the USA for dialysis patients younger than 45 years is over 100 times that in the general population. Many but not all - studies have demonstrated that even mild renal insufficiency is an independent risk factor for $\mathrm{CVD}^{4,5}$. Unfortunately, to date there have been no large-scale studies demonstrating that intervention can reduce the high cardiovascular risk in renal patients. However, it seems likely that cardiovascular risk factors present the same health hazards to patients with CRF as they do to the general population, and therefore that intervention to modify the risks will be beneficial.

Several mechanisms may explain the association between renal disease and CVD. Atherosclerosis will affect the main renal arteries in parallel with arteries elsewhere in the body, limiting blood flow and resulting in ischaemic damage. It is plausible that the same risk factors may promote intrarenal vascular disease, directly damaging glomeruli and tubules. It has also become apparent that glomerulosclerosis and atherosclerosis share a common pathophysiological aetiology ${ }^{6}$. Both processes are known to result from endothelial injury, macrophage infiltration, hyperlipoproteinaemia and

\section{Key Points}

Cardiovascular disease is common in patients with chronic renal failure (CRF)

\section{Atherosclerosis and glomerulosclerosis share a common aetiology and have common risk factors \\ Therapies which slow the progression of CRF are likely to improve cardiovascular outcome, and vice versa \\ Antihypertensive medication (including angiotensin-converting enzyme inhibitors and lipid lowering agents) and smoking cessation should be part of a comprehensive therapeutic approach to the patient with CRF}

KEY WORDS: CPD, atherosclerosis, cardiovascular disease, chronic renal failure, dyslipidaemia, glomerulosclerosis, hypertension, smoking 